\title{
Role of PCR in the diagnosis of pertussis infection in infants: 5 years' experience of provision of a same-day real-time PCR service in England and Wales from 2002 to 2007
}

\author{
Correspondence \\ Norman K. Fry \\ norman.fry@hpa.org.uk
}

Received 27 January 2009

Accepted 9 April 2009

\author{
Norman K. Fry, ${ }^{1}$ John Duncan, ${ }^{1}$ Karen Wagner, ${ }^{2}$ Oceanis Tzivra, ${ }^{1}$ \\ Nita Doshi, ${ }^{1}$ David J. Litt, ${ }^{1}$ Natasha Crowcroft, ${ }^{2}+$ Elizabeth Miller, ${ }^{2}$ \\ Robert C. George ${ }^{1}$ and Timothy G. Harrison ${ }^{1}$ Colindale Avenue, London, UK
${ }^{2}$ Immunisation, Hepatitis and Blood Safety Department, Health Protection Agency Centre for Infections, 61 Colindale Avenue, London, UK \\ ${ }^{1}$ Respiratory and Systemic Infection Laboratory, Health Protection Agency Centre for Infections, 61
}

As part of an enhanced surveillance programme for pertussis in England and Wales, a real-time PCR service for the detection of Bordetella pertussis was introduced for infants aged $\leqslant 6$ months admitted to a paediatric intensive care unit or paediatric ward with a respiratory illness compatible with pertussis. Two real-time fluorescent resonance energy transfer hybridization probe LightCycler (Roche Diagnostics) PCR assays were used. One (designed in-house) targeted the pertussis toxin $\mathrm{S} 1$ promoter ( $p t x A-\mathrm{pr}$ ), and included an internal process control to test for sample inhibition and reagent performance. The other (already published) targeted the insertion element IS 481. The analytical sensitivities of the assays were 100 and $10 \mathrm{fg}$ per reaction for the ptxA-pr and IS 481 PCRs, respectively. The ptxA-pr assay was specific for $B$. pertussis, whilst the IS 481 PCR also showed some cross-reactivity with Bordetella holmesii and the type strain of Bordetella parapertussis. From April 2002 to March 2007, 848 samples were received from 774 patients and DNA was extracted. Of 824 samples that were suitable for testing, $183(22.2 \%)$ had evidence of Bordetella infection (18.9\% ptxA-pr and IS481; 3.3\% IS481 only), 621 (75.4\%) were negative and $20(2.4 \%)$ were inhibitory for the PCR. Within the targeted age group of $\leqslant 6$ months, most patients (130/138) with evidence of Bordetella spp. by PCR were $\leqslant 3$ months old. The overall percentage increase in laboratory-confirmed cases due to PCR compared with culture for the 5 year period described ranged from 9 to $26 \%$ per year (mean $19 \%$ ). Real-time PCR is an invaluable tool both for enhanced epidemiological surveillance and for the provision of a rapid diagnosis of pertussis where results can affect patient and contact management.

\section{INTRODUCTION}

Infection with Bordetella pertussis (pertussis or whooping cough) causes significant morbidity and mortality and is one of the least-well-controlled vaccine-preventable diseases (Ward et al., 2005). Pertussis remains an important cause of infant death worldwide and is still of concern, even in countries with high vaccination coverage (WHO, 2005). Mild pertussis may be unrecognized in adults, and such adults may act as a source of infection for those most at risk (infants <1 year) (Nelson, 1978; Herwaldt, 1991;

tPresent address: Ontario Agency for Health Protection and Promotion, 415 Yonge Street, Suite 1601, Toronto, ON M5B 2E, Canada.

Abbreviations: IPC, internal process control; NPA, nasopharyngeal aspirate; PNS, pernasal swab; WHO, World Health Organization.
Crowcroft et al., 2003; Senanayake, 2007). In England and Wales, pertussis is a notifiable disease, and the level of notifications fell from 883 in 2002 to a historic low of 409 in 2003. Since then, numbers have increased to between 500 and 600 per year in 2004-2006, but in 2007 this number rose to 1089 (HPA, 2008). However, the main increase in notifications has been in the 15 years and older age group, and is probably due to improved ascertainment due to greater awareness and a consequent increase in laboratory testing (Harnden et al., 2006; Litt et al., 2006; HPA, 2007). Laboratory confirmation of pertussis infection can be made by isolation of the causative organism, $B$. pertussis; demonstration of the presence of genomic DNA from the organism in clinical samples, typically nasopharyngeal aspirates (NPAs) and pernasal swabs (PNSs), by 
PCR; and estimation of the levels of specific antibodies to pertussis by serological or oral fluid assays (Giammanco et al., 2003a, b; Litt et al., 2006; André et al., 2008). Whilst culture of $B$. pertussis is to be encouraged and is traditionally viewed as the definitive diagnosis of pertussis, it can be slow and of varying, often low, sensitivity (Dragsted et al., 2004; Bamberger et al., 2005). In the absence of a vaccine history, serological tests - or more recently oral fluid antibody tests - cannot usually distinguish between recent vaccination against pertussis and recent infection, although they are still useful in providing a retrospective diagnosis, particularly for older children and adults, and can be more sensitive than culture (Kerr \& Matthews, 2000; Litt et al., 2006).

The utility of PCR for the diagnosis of pertussis is now well established and has been shown to have improved sensitivity over culture (Grimprel et al., 1993; He et al., 1993; Muyldermans et al., 2005). In addition, improvements in nucleic acid extraction and real-time PCR technologies have resulted in shorter assay turnaround times (Reischl et al., 2001a; Muyldermans et al., 2005). Various genomic targets have been described, including those targeting the promoter region of the gene encoding the pertussis toxin S1 subunit (ptxA) (Houard et al., 1989; Mastrantonio et al., 1996), the insertion element IS481 (Glare et al., 1990), the adenylate cyclase-encoding gene (cyaA) (Douglas et al., 1993) and a region upstream of the outer-membrane porin gene (Li et al., 1994). Several of these assays have been implemented on real-time platforms (Muyldermans et al., 2005).

An enhanced surveillance scheme for pertussis infection (based on laboratory-confirmed cases with epidemiological follow-up) was established in 1994 for England and Wales by the former Public Health Laboratory Service (Van Buynder et al., 1999) and is now provided by the Health Protection Agency. The purpose of this scheme is to monitor pertussis infection and vaccine efficacy in order to enable informed decisions to be made with respect to vaccine policy (e.g. to assess the need for booster vaccinations). As part of this scheme, in addition to capturing data from culture-confirmed cases, new diagnostic methods (serodiagnosis and PCR detection) were introduced by the Respiratory and Systemic Infection Laboratory and officially launched in April 2002.

This study reports on the experience of a reference laboratory using a real-time PCR assay for B. pertussis adapted from the previously described block-based thermocycler PCR assay using detection of products by gel electrophoresis (Fry et al., 2004). This real-time PCR service was applied to clinical specimens as a same-day service for pertussis diagnosis in infants ( $\leqslant 6$ months) over a 5 year period from April 2002 to March 2007. Where other samples were submitted from these patients (i.e. $B$. pertussis isolates for confirmation), these data are also presented.

\section{METHODS}

Patients, specimens and inclusion criteria. The data presented are from specimens received between 1 April 2002 and 31 March 2007. Inclusion criteria for the offered B. pertussis PCR service were: NPAs or PNSs (collected with a fine, flexible, twisted Nichrome wire shaft swab and rayon bud) not previously used for culture, from infants $\leqslant 6$ months of age with respiratory illness compatible with pertussis infection, admitted to paediatric intensive care units or paediatric wards. Samples fulfilling these criteria received by 10 a.m. (Monday to Friday) were processed and reported the same day. Specimens and resulting data from outside these criteria (not routinely tested on the same day, but in the next available run) are noted as such in the text. Where available, relevant data from additional samples from these patients (i.e. putative B. pertussis isolates for confirmation and serotyping) are also presented. Clinical samples submitted to the PCR service were extracted as described below and our laboratory did not attempt to isolate B. pertussis directly from these samples. The identity of putative $B$. pertussis isolates submitted for confirmation and serotyping was established using standard methods (Fry et al., 2004).

DNA extraction. The reference strains B. pertussis, Bordetella avium, Bordetella bronchiseptica, Bordetella hinzii, Bordetella holmesii, Bordetella parapertussis, Bordetella trematum, Bordetella petrii and 'Bordetella ansorpii' were obtained from the National Collection of Type Cultures (UK) and the Culture Collection, University of Göteborg (Sweden). Genomic DNA used for positive controls or specificity experiments from members of the genus Bordetella (Von Wintzingerode et al., 2001) was prepared as described previously (Fry et al., 2004). Previously extracted genomic DNA from other genera and species (Bartonella birtlesii, Bartonella bacilliformis, Chlamydia spp., Chlamydiophyla spp., Citrobacter spp., Corynebacterium diphtheriae, Coxiella spp., Enterococcus faecalis, Escherichia coli, Haemophilus influenzae, Klebsiella spp., Legionella pneumophila, Legionella bozemanae, Neisseria meningitidis, Mycoplasma orale, Mycoplasma pneumoniae, Proteus spp., Pseudomonas aeruginosa, Ureaplasma spp., Staphylococcus aureus, Streptococcus anginosus, Streptococcus pyogenes, Streptococcus intermedius, Lancefield group G Streptococcus, Lancefield group B Streptococcus, Streptococcus salivarius, Streptococcus bovis, Streptococcus gordonii, Streptococcus oralis and Gemella haemolysins) were obtained from the authors' laboratory (Respiratory and Systemic Infection Laboratory). Genomic DNA suitable as template for the $B$. pertussis PCR assays was extracted from clinical specimens, including respiratory samples (usually NPAs and PNSs) or tissue samples using a QiaAmp DNA mini kit (Qiagen) or MagNaPure Compact (Roche) according to the instructions of the manufacturer. To control for possible contamination of the extraction procedure, a negative control comprising $200 \mu \mathrm{l}$ PBS was subjected to the same extraction procedure with each batch of clinical samples. Typically, a volume of 200-400 $\mu$ NPA was extracted. Where the starting volume was less than $200 \mu$ l, it was made up to $200 \mu \mathrm{l}$ with nuclease-free water (Sigma). For PNSs, the flexible wire shaft was cut approximately $1.5 \mathrm{~cm}$ from the swab tip with sterile scissors and the swab was vortexed in $200 \mu \mathrm{l}$ PBS, expressed on the side of the tube and removed using sterile forceps prior to extraction of the solution. DNA extracts were eluted in $100 \mu$ nuclease-free water and were either assayed immediately or stored at $4{ }^{\circ} \mathrm{C}$. Longterm storage of extracts was at $-25{ }^{\circ} \mathrm{C}$ or below. Extracted clinical samples were tested at neat, $1: 10$ and $1: 100$ dilutions in nuclease-free water (Sigma).

Construction of an internal process control (IPC) for detection of inhibition. The presence or absence of PCR inhibitors and the performance of the PCR assay in extracted specimens were determined using an IPC. The IPC was constructed to contain bacteriophage $\lambda$ DNA within sequences homologous to the two 
primers used in the $p t x A$-pr assay. Initially, a PCR product was amplified using $\lambda$ DNA as a template, and two primers specifically designed to incorporate both $\lambda$ DNA and the ptxA-pr assay primer sequences (Table 1). This product was then cloned into a TOPO plasmid vector (TOPO TA cloning kit; Invitrogen) and the construct was transformed into competent Escherichia coli One Shot TOP10 cells (Invitrogen). Transformants were subcultured on Luria-Bertani medium containing $50 \mu \mathrm{g}$ ampicillin $\mathrm{ml}^{-1}$ (Sigma), and purified plasmid DNA was prepared using a QIAprep miniprep kit (Qiagen). Plasmid preparations were linearized using a restriction endonuclease with a single site in the vector and none within the cloned region (e.g. XbaI), and re-precipitated with $3 \mathrm{M}$ sodium acetate $(\mathrm{pH} 5.2)$ at a final concentration of $0.3 \mathrm{M}$ and ethanol (Sambrook \& Russell, 2001). Linearized plasmid IPC was titrated in the ptxA-pr assay to achieve a crossing point of approximately 33 cycles ( $\sim 5$ copies, 0.2 fg per reaction mix) with probes IPC-DNR and IPC-ACR (Table 1) targeted against the internal $\lambda$ DNA. A linear (nonexponential) result in the absence of any positive signal with $p t x A$ or IS481 was scored as inhibitory.

\section{B. pertussis LightCycler PCR assays targeting the B. pertussis} toxin promoter together with an IPC and targeting the insertion element IS481. A consensus (dual-target) PCR approach was used; LightCycler (Roche Diagnostics) assays for two gene targets were designed using fluorescence resonance energy transfer hybridization probes for confirmation of products. The donor fluorophore, fluorescein, was used to label one of each pair of probes at the $3^{\prime}$ terminus, and the acceptor fluorophore, LC-Red 640 or LC-Red 705, was used to label the other at the $5^{\prime}$ terminus (Table 1 ). The first assay was designed in house and targeted the pertussis toxin S1 (ptxA) promoter region ( $p t x A$-pr PCR). This assay comprised one pair of primers targeting the $B$. pertussis ptxA promoter and two pairs of hybridization probes, one pair designed to target a region internal to the $p t x A$-pr product, and one pair specific for the $\lambda$ sequence within the IPC as described above (Table 1). The second assay (IS481 PCR), described by Reischl et al. (2001a), comprised one pair of primers targeting the insertion sequence (IS481) of B. pertussis and one pair of hybridization probes. This PCR targeted the insertion element IS481 (which occurs in B. pertussis, B. holmesii and some strains of $B$. bronchiseptica and B. parapertussis) (Reischl et al., 2001a, b; Kösters et al., 2002). All reaction mixtures were in a total volume of $20 \mu \mathrm{l}$ and comprised $1 \times$ LightCycler FastStart DNA master hybridization probes mix (Roche Diagnostics), $0.5 \mu \mathrm{M}$ each primer, $0.25 \mu \mathrm{M}$ each probe and $1 \mathrm{U}$ heat-labile uracil-DNA glycosylase (Roche). The two assays included final concentrations of $4.0 \mathrm{mM} \mathrm{MgCl}$ (Roche) for the $p t x A$ pr assay and $3.0 \mathrm{mM} \mathrm{MgCl}_{2}$ for the IS481 assay. The $p t x A$-pr assay also contained approximately 45 copies $(0.2 \mathrm{fg})$ of the ptxA-pr IPC. Amplification and detection were performed with a LightCycler (using LightCycler software version 5.32). The cycling conditions used were based on those described by Reischl et al. (2001a) with the addition of a melt and cooling step: initial denaturation for $10 \mathrm{~min}$ at $95{ }^{\circ} \mathrm{C}$, followed by 50 cycles of denaturation for $10 \mathrm{~s}$ at $95{ }^{\circ} \mathrm{C}$, annealing for $10 \mathrm{~s}$ at $50{ }^{\circ} \mathrm{C}$ and extension for $20 \mathrm{~s}$ at $72{ }^{\circ} \mathrm{C}$; a melt step of $95{ }^{\circ} \mathrm{C}$ for $0 \mathrm{~s}, 40{ }^{\circ} \mathrm{C}$ for $30 \mathrm{~s}$ and $95{ }^{\circ} \mathrm{C}$ for $0 \mathrm{~s}$ (with a ramping rate of $0.1{ }^{\circ} \mathrm{C} \mathrm{s}^{-1}$ ); and a final cooling step of $40{ }^{\circ} \mathrm{C}$ for $30 \mathrm{~s}$. Except where stated otherwise, all ramping rates were maximal $\left(20^{\circ} \mathrm{C} \mathrm{s}^{-1}\right)$. Fluorescence was measured using the single acquisition mode during the annealing step or continuous mode in the third segment of the melt. All extracted specimens were tested in the LightCycler PCR assays using $5 \mu \mathrm{l}$ undiluted extract, together with $5 \mu \mathrm{l}$ of $1: 10$ and $1: 100$ dilutions (in nuclease-free water). Positive controls of purified B. pertussis DNA were included, using at least four dilutions for each assay $(100,10,1$ and $0.1 \mathrm{pg}$ for the ptxA-pr assay, and 10, 1, 0.1 and $0.01 \mathrm{pg}$ for the IS481 LightCycler assay) in order to calculate a standard curve. Additional controls included with each batch of samples tested comprised $5 \mu \mathrm{l}$ undiluted extract from a negative control of PBS (extracted as described above) and $5 \mu$ l nuclease-free water (no added DNA).

Interpretation of LightCycler PCR results. Usually, analyses were performed using the automated (default) method with colour compensation for the $p t x A-p r$ and IPC results. If required, results were also examined using the fitted-points method. For reporting purposes, the real-time PCR results for B. pertussis were divided into five classes: (i) $B$. pertussis positive, defined by a positive amplification signal in both the ptxA-pr and IS481 PCRs; (ii) B. pertussis negative, defined by the absence of a positive amplification signal in both the ptxA-pr and IS481 PCRs, together with a positive signal with the IPC;

Table 1. Oligonucleotides used in this study

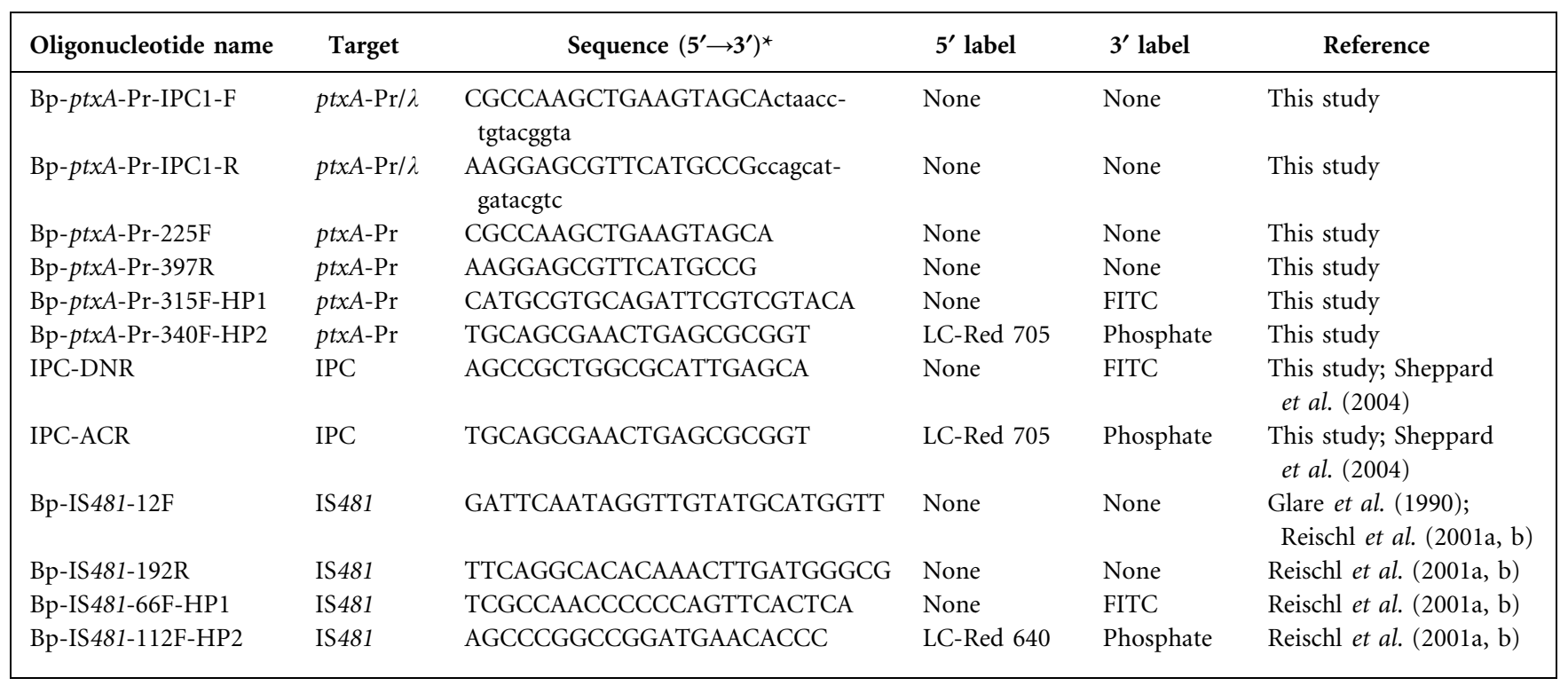

FITC, Fluorescein isothiocyanate.

${ }^{*}$ The pertussis toxin promoter sequence is in upper case and the $\lambda$ sequence in lower case. 
(iii) Bordetella spp. positive, defined by a positive amplification signal in the IS481 PCR only; (iv) inhibitory, defined by a non-exponential/ linear amplification signal with the IPC; and (v) equivocal, defined by a positive amplification signal in the ptxA-pr PCR only. It should be noted that, due to the greater sensitivity of the IS 481 assay, a positive signal in this assay without a corresponding signal in the ptxA-pr assay could be due to either very low amounts of $B$. pertussis or to the presence of another Bordetella species such as B. holmesii (Reischl et al., 2001b), B. bronchiseptica or B. parapertussis. In equivocal cases, further specimens for PCR and/or serological investigation were requested if appropriate.

\section{RESULTS AND DISCUSSION}

\section{PCR sensitivity and specificity}

The analytical sensitivities of the Bordetella PCR LightCycler assays were determined to be 100 and $10 \mathrm{fg}$ per reaction for the ptxA-pr and the IS481 PCRs, respectively. Although the sensitivity of the $p t x A$-pr could reach $10 \mathrm{fg}$, because this level of detection was not consistently achieved, the sensitivity of this assay was taken to be $100 \mathrm{fg}$ for reporting purposes. Of the nine species within the genus Bordetella, the ptxA-pr assay was specific for B. pertussis (Table 2).

The IS481 assay as used here was originally described as specific for B. pertussis (Reischl et al., 2001a), using a number of strains belonging to five Bordetella species and 80 Gram-positive and Gram-negative organisms from other genera. It has since been shown to give a positive signal with $B$. holmesii, and some strains of $B$. parapertussis and $B$. bronchiseptica. We confirmed the cross-reactivity with $B$.

Table 2. Specificity of PCR assays for members of the genus Bordetella

\begin{tabular}{|lcc|}
\hline Species & ptxA-pr & IS481 \\
\hline B. pertussis NCTC $10739^{\mathrm{T}}$ & + & - \\
B. pertussis NCTC 8189 & + & + \\
B. avium NCTC $12033^{\mathrm{T}}$ & - & - \\
B. avium NCTC 12034 & - & - \\
B. bronchiseptica NCTC $452^{\mathrm{T}}$ & - & - \\
B. bronchiseptica NCTC 458 & - & - \\
B. parapertussis NCTC $5952^{\mathrm{T}}$ & - & - \\
B. parapertussis NCTC 10853 & - & - \\
B. hinzii NCTC $13199^{\mathrm{T}}$ & - & - \\
B. hinzii NCTC 13200 & - & + \\
B. holmesii NCTC $12912^{\mathrm{T}}$ & - & - \\
B. holmesii NCTC 13202 & - & - \\
B. trematum NCTC $12995^{\mathrm{T}}$ & - & - \\
B. trematum NCTC 13203 & - & - \\
B. petrii CCUG $43448^{\mathrm{T}}$ & - & \\
B. petrii NCTC 13363 & - & - \\
'B. ansorpii' NCTC $13364^{\mathrm{T}}$ & - & \\
\hline
\end{tabular}

CCUG, Culture Collection University of Göteborg; NCTC, National Collection of Type Cultures.

${ }^{\star}$ Crossing point value $>40$. holmesii and also with the type strain of $B$. parapertussis (although in the latter case this had a crossing point of $>40$ cycles). We extended the specificity experiments in this study by first (re)-testing purified DNA from strains belonging to all nine described species of Bordetella and from other genera. Neither assay gave a positive amplification signal with any member of a non-Bordetella genus tested. The type strain of $B$. pertussis, which has been found to be atypical in many of its characteristics compared with other B. pertussis strains (Packard et al., 2004), did not give an amplification signal with the IS481 assay, presumably due to variation in the primer or probe sites.

\section{Patients, specimens and PCR results}

During the study period, 848 samples from 774 patients were submitted for analysis. Single specimens were obtained from the majority $(716 / 774,92.5 \%)$ of patients, followed by two specimens per patient (52/774, 6.7\%). Occasionally, multiple samples were received: three specimens from each of four patients, and seven and nine postmortem samples from two patients. Details of the numbers of patients by age and the results obtained by PCR are shown in Table 3. The majority (584/774, $75.5 \%$ ) belonged to the target group of $\leqslant 6$ months. The gender was stated for $721 / 774$ patients $(93.4 \%)$ from whom samples were received and the ratio was 1.03:1 for males: females. The majority of samples submitted were respiratory samples comprising NPAs (489 samples, 57.7\%,), PNSs (249 samples, $29.4 \%$ ), endotracheal secretions (24 samples, $2.8 \%$ ); bronchoalveolar lavages (30 samples, $3.5 \%$ ); throat and nose swabs (15 samples, $1.8 \%$ ) and sputum (5 samples, $0.6 \%)$. Other specimen types submitted included tissue (13 samples, $1.5 \%$ ), cerebrospinal fluid (1 sample, $0.01 \%)$, EDTA blood or sera (7 samples, $0.8 \%$ ) and some samples whose nature or site was not specified (scored as not known) (15 samples, $1.8 \%$ ).

\section{Detection of Bordetella by PCR}

We used a consensus approach together with an internal control in order to provide greater confidence in the results

Table 3. Results of testing 774 patients by $B$. pertussis PCR from April 2002 to March 2007

\begin{tabular}{|lcc|}
\hline Age group & No. of patients & $\begin{array}{c}\text { No. of patients with } \\
\text { evidence of Bordetella spp. } \\
\text { by PCR }(\%)\end{array}$ \\
\hline$\leqslant 6$ months & 584 & $138(23.6)$ \\
$7-11$ months & 52 & $4(7.7)$ \\
$1-10$ years & 82 & $19(23.2)$ \\
$11-20$ years & 19 & $3(15.8)$ \\
$21-66$ years & 25 & $2(8.0)$ \\
Unknown & 12 & $2(1.7)$ \\
Total & 774 & $168(21.7)$ \\
\hline
\end{tabular}


obtained. Two independent targets were used ( $p t x A$-pr and IS481) and an IPC was used to test for the presence of PCR inhibitors and to confirm the integrity of the assay. From April 2002 to March 2007, 848 samples were received from 774 patients for investigation by $B$. pertussis PCR. Twentyfour samples $(2.8 \%)$ were not tested by PCR for various reasons, e.g. unsuitable sample due to age of sample, duplicate or incorrect sample type such as EDTA blood or serum. Of the 824 samples tested, 183 (22.2\%) had evidence of Bordetella spp. (18.9\% ptxA-pr and IS481; $3.2 \%$ IS481 only), whilst $621(75.4 \%)$ were negative and $20(2.4 \%)$ were inhibitory. The inhibitory samples included 14/489 NPAs, 5/ 249 PNSs and $1 / 30$ bronchoalveolar lavages, but no statistically significant association of inhibition and sample type was found. Some samples contained PCR inhibitors resulting in either total (scored as inhibitory) or partial inhibition. Typically, dilution of the extracted DNA removed or reduced the inhibitory effect allowing the sample to be scored as negative or positive, but with a concomitant reduction in the sensitivity of the assay. The crossing point values for samples positive for both targets were usually at least 3.3 cycles higher in the IS481 assay corresponding to at least a tenfold increase in sensitivity. In samples positive for the IS 481 only, crossing points were usually observed after 35 cycles. In one case where sequential samples were obtained from an infant transferred between hospitals, the earlier specimen taken prior to antibiotic therapy was both $p t x A$-pr and IS481 positive, whilst the later one was IS481 positive only.

In special circumstances, post-mortem samples from tissue samples (including lung, liver, kidney and spleen) were investigated from four patients, one of whom was a case of sudden infant death syndrome (SIDS). One (non-SIDS) patient was PCR positive ( $p t x A$-pr and IS481). Blood and sera samples are considered inappropriate samples for $B$. pertussis PCR investigation and these samples were not tested by this method; however, estimation of antipertussis toxin IgG antibodies in such samples was undertaken if appropriate (i.e. if the patient had not been vaccinated recently). For PCR, respiratory samples were either processed immediately or held at $-80{ }^{\circ} \mathrm{C}$ before genomic DNA extraction.

\section{Results from patients' samples submitted in addition to those investigated by PCR}

Putative B. pertussis isolates (for confirmation and/or serotyping) were received from a number of patients tested by PCR. Of $36 \mathrm{~B}$. pertussis PCR-positive patients, isolates from 17 of these patients were confirmed as $B$. pertussis by culture. There was $1 \mathrm{~B}$. pertussis culture-positive sample from 30 patients that were PCR negative for B. pertussis, but the specimen submitted (a throat swab) to our laboratory for PCR from this patient was one not considered suitable for the collection of B. pertussis.

The PCR results from this service, together with confirmed B. pertussis isolates and serological data, comprise the laboratory-confirmed cases of pertussis reported to the pertussis enhanced surveillance scheme. Despite the limitations of the PCR service described, i.e. restricted target age and service not offered out of regular working hours, we believe it has provided real benefits in a number of ways, including a more rapid turnaround time. The previous block-based methodology took a mean time of $3 \mathrm{~h}$ per PCR run, making the whole assay process from receipt of specimen to reporting of result difficult to fit into a working day. In addition, although the mean number of days from sample collection to receipt was 3.6 days, the modal number of days was 1 . For primary isolation, $B$. pertussis can grow on Bordetella-selective medium (charcoal blood agar with cephalexin) within 3 days, but 57 days may be required. Moreover, it is recommended that inoculated plates are incubated for 7 days before being discarded as negative (Matthews, 1997). For samples delivered to us within $24 \mathrm{~h}$ of collection, a result on the same day as receipt was routinely provided, offering a real opportunity to affect patient management. Inclusion of an IPC prevents the reporting of false-negative results and effectively controls the PCR process. The rationale for using a consensus (two-target) approach was to ensure complete confidence in the results obtained. However, the superior sensitivity of the IS481 assay due to the presence of IS481 in multiple copies on the B. pertussis genome has led to its widespread use, usually as a single-target assay. The presence of Bordetella species other than B. pertussis in respiratory samples submitted for $B$. pertussis PCR cannot be ruled out, but to date the presence and frequency of these other species, which may give cross-reactivity using IS481 as a target, appear to be low. The recovery of $B$. holmesii from NPA specimens of otherwise healthy young persons with a cough has been demonstrated (Yih et al., 1999), but B. holmesii DNA was not found in DNA extracts of NPA swabs from Finnish and Dutch patients with suspected pertussis (Antila et al., 2006). The non-specific nature of IS 481 as a target for $B$. pertussis sequences has recently led to the description of new targets for specific detection of this species (Probert et al., 2008). Concern has also been raised over false positives and/or contamination in laboratories performing PCR (Probert et al., 2008). During the course of this study, our laboratory participated in an external quality assessment for the molecular detection of $B$. pertussis in European laboratories (Muyldermans et al., 2005). No false-positive results were reported by any of the six laboratories, although misidentification of $B$. holmesii and $B$. bronchiseptica for the reasons described above was observed. This highlights the value of such external quality assessment schemes and the need for all laboratories involved in PCR testing to participate.

The added value of the PCR can be measured in different ways, but all make assumptions. If one assumes that cases of suspected pertussis would be missed (by lack of laboratory confirmation) in the absence of the $B$. pertussis PCR, the percentage increase due to the PCR, compared 
with culture (in the $\leqslant 6$ months group) ranged from 9 to $26 \%$ (mean 19\%) from 2002 to 2007. Although in some cases B. pertussis was isolated after the specimen was found to be PCR positive, and it could be argued that availability of PCR testing for $B$. pertussis may lead to an overall decrease in attempts to culture, the rapid nature of the PCR test provides a considerable advantage. The detection of PCR positives in the older age groups has led us to increase the age range to $\leqslant 1$ year (from April 2007), although the majority of cases are still in the $\leqslant 6$ months group.

In conclusion, real-time PCR is an invaluable tool both for enhanced epidemiological surveillance and for the provision of a rapid diagnosis of pertussis where results can affect patient (and contact) management. Together with serological results, and isolation and confirmation of the identity of putative $B$. pertussis isolates, these data are invaluable in enabling informed decisions to be made with respect to the vaccination programme for pertussis. During the course of this study, our laboratory also assisted in a number of potential outbreak/cross-infection investigations in healthcare workers on paediatric intensive care units and children's wards by providing rapid screening of healthcare workers and infants. We demonstrated an increase in the detection rate of pertussis infection using PCR, compared with culture, in a limited target age group. If PCR detection were to be used routinely for all ages, then more accurate estimates of pertussis infection should be possible. In the future, we anticipate that, increasingly, regional and hospital laboratories equipped for PCR will be able to test for B. pertussis and B. parapertussis using inhouse published or commercial assays. Reference laboratories could provide confirmation of these results, using a species-specific target (as illustrated for B. pertussis in this study) and additional typing data, such as multi-locus variable-number tandem repeat analysis directly from PCRpositive material (Alexander et al., 2008; Litt et al., 2009).

\section{ACKNOWLEDGEMENTS}

We thank the hospitals and microbiology laboratories for submission of specimens, the staff of the Atypical Pneumonia Unit (Health Protection Agency Centre for Infections) for their technical support and Udo Reischl for helpful advice concerning the IS481 assay.

\section{REFERENCES}

Alexander, E. M., Travis, S., Booms, C., Kaiser, A., Fry, N. K., Harrison, T. G., Ganpot, B. \& Klein, J. L. (2008). Pertussis outbreak on a neonatal unit: identification of a healthcare worker as the likely source. J Hosp Infect 69, 131-134.

André, P., Caro, V., Njamkepo, E., Wendelboe, A. M., Van Rie, A. \& Guiso, N. (2008). Comparison of serological and real-time PCR assays to diagnose Bordetella pertussis infection in 2007. J Clin Microbiol 46, 1672-1677.

Antila, M., He, Q., de Jong, C., Aarts, I., Verbakel, H., Bruisten, S., Keller, S., Haanperä, M., Mäkinen, J. \& other authors (2006). Bordetella holmesii DNA is not detected in nasopharyngeal swabs from Finnish and Dutch patients with suspected pertussis. J Med Microbiol 55, 1043-1051.

Bamberger, E., Lahat, N., Gershtein, V., Gershtein, R., Benilevi, D., Shapiro, S., Kassis, I., Rubin, L. \& Srugo, I. (2005). Diagnosing pertussis: the role of polymerase chain reaction. Isr Med Assoc J 7, 351-354.

Crowcroft, N. S., Booy, R., Harrison, T., Spicer, L., Britto, J., Mok, Q., Heath, P., Murdoch, I., Zambon, M. \& other authors (2003). Severe and unrecognised: pertussis in UK infants. Arch Dis Child 88, 802806.

Douglas, E., Coote, J. G., Parton, R. \& McPheat, W. (1993). Identification of Bordetella pertussis in nasopharyngeal swabs by PCR amplification of a region of the adenylate cyclase gene. $J$ Med Microbiol 38, 140-144.

Dragsted, D. M., Dohn, B., Madsen, J. \& Jensen, J. S. (2004). Comparison of culture and PCR for detection of Bordetella pertussis and Bordetella parapertussis under routine laboratory conditions. J Med Microbiol 53, 749-754.

Fry, N. K., Tzivra, O., Li, Y. T., McNiff, A., Doshi, N., Maple, P. A. C., Crowcroft, N. S., Miller, E., George, R. C. \& Harrison, T. G. (2004). Laboratory diagnosis of pertussis infections: the role of PCR and serology. J Med Microbiol 53, 519-525.

Giammanco, A., Taormina, S., Chiarini, A., Dardanoni, G., Stefanelli, P., Salmaso, S. \& Mastrantonio, P. (2003a). Analogous IgG subclass response to pertussis toxin in vaccinated children, healthy or affected by whooping cough. Vaccine 21, 1924-1931.

Giammanco, A., Chiarini, A., Maple, P. A. C., Andrews, N., Pebody, R., Gay, N., Ölander, R. M., Fivet-Groyne, F., Baron, S. \& other authors (2003b). European Sero-Epidemiology Network: standardisation of the assay results for pertussis. Vaccine 22, 112-120.

Glare, E. M., Paton, J. C., Premier, R. R., Lawrence, A. J. \& Nisbet, I. T. (1990). Analysis of a repetitive DNA sequence from Bordetella pertussis and its application to the diagnosis of pertussis using the polymerase chain reaction. J Clin Microbiol 28, 1982-1987.

Grimprel, E., Bégué, P., Anjak, I., Betsou, F. \& Guiso, N. (1993). Comparison of polymerase chain reaction, culture, and Western immunoblot serology for diagnosis of Bordetella pertussis infection. J Clin Microbiol 31, 2745-2750.

Harnden, A., Grant, C., Harrison, T., Perera, R., Brueggemann, A. B., Mayon-White, R. \& Mant, D. (2006). Whooping cough in school age children with persistent cough: prospective cohort study in primary care. BMJ 333, 174-177.

He, Q., Mertsola, J., Soini, H., Skurnik, M., Ruuskanen, O. \& Viljanen, M. K. (1993). Comparison of polymerase chain reaction with culture and enzyme immunoassay for diagnosis of pertussis. J Clin Microbiol 31, 642-645.

Herwaldt, L. A. (1991). Pertussis in adults. What physicians need to know. Arch Intern Med 151, 1510-1512.

Houard, S., Hackel, C., Herzog, A. \& Bollen, A. (1989). Specific identification of Bordetella pertussis by the polymerase chain reaction. Res Microbiol 140, 477-487.

HPA (2007). Health Protection Report 2, 9-10. London: Health Protection Agency. http://www.hpa.org.uk/hpr/archives/2008/hpr4808. pdf

HPA (2008). Statutory Notifications of Infectious Diseases (NOIDs) Annual Totals 1994 to 2007 - England and Wales. London: Health Protection Agency. http://www.hpa.org.uk/web/HPAweb\&HPAweb Standard/HPAweb_C/1195733756346

Kerr, J. R. \& Matthews, R. C. (2000). Bordetella pertussis infection: pathogenesis, diagnosis, management, and the role of protective immunity. Eur J Clin Microbiol Infect Dis 19, 77-88. 
Kösters, K., Reischl, U., Schmetz, J., Riffelmann, M. \& Von Konig, C. H. W. (2002). Real-time LightCycler PCR for detection and discrimination of Bordetella pertussis and Bordetella parapertussis. J Clin Microbiol 40, 1719-1722.

Li, Z., Jansen, D. L., Finn, T. M., Halperin, S. A., Kasina, A., O'Connor, S. P., Aoyama, T., Manclark, C. R. \& Brennan, M. J. (1994). Identification of Bordetella pertussis infection by shared-primer PCR. J Clin Microbiol 32, 783-789.

Litt, D. J., Samuel, D., Duncan, J., Harnden, A., George, R. C. \& Harrison, T. G. (2006). Detection of anti-pertussis toxin IgG in oral fluids for use in diagnosis and surveillance of Bordetella pertussis infection in children and young adults. J Med Microbiol 55, 12231228.

Litt, D. J., Neal, S. E. \& Fry, N. K. (2009). Changes in genetic diversity of the Bordetella pertussis population in the United Kingdom between 1920 and 2006 reflect vaccination coverage and the emergence of a single dominant clonal type. J Clin Microbiol 47, 680-688.

Mastrantonio, P., Stefanelli, P. \& Giuliano, M. (1996). Polymerase chain reaction for the detection of Bordetella pertussis in clinical nasopharyngeal aspirates. J Med Microbiol 44, 261-266.

Matthews, R. (1997). The diagnosis of pertussis infections: a recurring challenge. Publ Health Lab Serv Microbiol Digest 14, 79-84.

Muyldermans, G., Soetens, O., Antoine, M., Bruisten, S., Vincart, B., Doucet-Populaire, F., Fry, N. K., Olcén, P., Scheftel, J. M. \& other authors (2005). External quality assessment for molecular detection of Bordetella pertussis in European laboratories. J Clin Microbiol 43 , 30-35.

Nelson, J. D. (1978). The changing epidemiology of pertussis in young infants. The role of adults as reservoirs of infection. Am J Dis Child 132, 371-373.

Packard, E. R., Parton, R., Coote, J. G. \& Fry, N. K. (2004). Sequence variation and conservation in virulence-related genes of Bordetella pertussis isolates from the UK. J Med Microbiol 53, 355-365.

Probert, W. S., Ely, J., Schrader, K., Atwell, J., Nossoff, A. \& Kwan, S. (2008). Identification and evaluation of new target sequences for specific detection of Bordetella pertussis by real-time PCR. J Clin Microbiol 46, 3228-3231.
Reischl, U., Burggraf, S., Leppmeier, B., Linde, H.-J. \& Lehn, N. (2001a). Rapid and specific detection of Bordetella pertussis in clinical specimens by LightCycler PCR. In Rapid Cycle Real-Time PCR: Methods and Applications, pp. 313-321. Edited by S. Meuer, C. Wittwer \& K.-I. Nakagawara. Berlin: Springer.

Reischl, U., Lehn, N., Sanden, G. N. \& Loeffelholz, M. J. (2001b). Real-time PCR assay targeting IS481 of Bordetella pertussis and molecular basis for detecting Bordetella holmesii. J Clin Microbiol 39, 1963-1966.

Sambrook, J. \& Russell, D. W. (2001). Molecular Cloning: a Laboratory Manual, 3rd edn, A8.12-14. Cold Spring Harbor, NY: Cold Spring Harbor Laboratory.

Senanayake, S. (2007). Pertussis in Australia today - a disease of adolescents and adults that can kill infants. Aust Fam Physician 36, 51-56.

Sheppard, C. L., Harrison, T. G., Morris, R., Hogan, A. \& George, R. C. (2004). Autolysin-targeted LightCycler assay including internal process control for detection of Streptococcus pneumoniae DNA in clinical samples. J Med Microbiol 53, 189-195.

Van Buynder, P. G., Owen, D., Vurdien, J. E., Andrews, N. J., Matthews, R. C. \& Miller, E. (1999). Bordetella pertussis surveillance in England and Wales: 1995-7. Epidemiol Infect 123, 403-411.

Von Wintzingerode, F., Schattke, A., Siddiqui, R. A., Rösick, U., Göbel, U. B. \& Gross, R. (2001). Bordetella petrii sp. nov., isolated from an anaerobic bioreactor, and emended description of the genus Bordetella. Int J Syst Evol Microbiol 51, 1257-1265.

Ward, J. I., Cherry, J. D., Chang, S.-J., Partridge, S., Lee, H., Treanor, J., Greenberg, D. P., Keitel, W., Barenkamp, S. \& other authors (2005). Efficacy of an acellular pertussis vaccine among adolescents and adults. N Engl J Med 353, 1555-1563.

WHO (2005). Pertussis vaccines - WHO position paper. Wkly Epidemiol Rec 80, 31-39.

Yih, W. K., Silva, E. A., Ida, J., Harrington, N., Lett, S. M. \& George, H. (1999). Bordetella holmesii-like organisms isolated from Massachusetts patients with pertussis-like symptoms. Emerg Infect Dis 5, 441-443. 